\title{
Protonic Conduction of Nanostructured Y-Doped $\mathrm{BaZrO}_{3}$
}

\author{
Hee Jung Park ${ }^{1}$ and Jong Wook Roh ${ }^{2}$ \\ ${ }^{1}$ Department of Advanced Materials Engineering, Daejeon University, 62 Daehak-ro, Dong-gu, Daejeon 34520, Republic of Korea \\ ${ }^{2}$ Materials Center, Samsung Advanced Institute of Technology, Samsung Electronics, 130 Samsung-ro, Suwon 16678, Republic of Korea \\ Correspondence should be addressed to Hee Jung Park; hjpark@dju.kr
}

Received 12 October 2016; Revised 19 November 2016; Accepted 8 December 2016

Academic Editor: Vincenzo Baglio

Copyright (C) 2016 H. J. Park and J. W. Roh. This is an open access article distributed under the Creative Commons Attribution License, which permits unrestricted use, distribution, and reproduction in any medium, provided the original work is properly cited.

\begin{abstract}
Nanostructured ionic conductors have recently attracted our attention due to the expectation that they may lead to new functionalities absent in microcrystalline conductors. In this study, nanostructured barium zirconate with perovskite crystal structure was prepared and its grain and grain boundary protonic conduction was investigated using ac impedance measurements as a function of temperature $\left(\mathrm{RT} \sim 400^{\circ} \mathrm{C}\right)$ and water partial pressure. The grain was highly conductive of protons, which is governed by the concentration of protonic defects at all temperatures. On the other hand, the grain boundary was not the preferred route for protonic conduction due to high resistance. However, enhanced protonic transport was observed at certain temperatures $\left(<100^{\circ} \mathrm{C}\right)$. The protonic resistivity below that temperature decreased with decreasing temperature, showing positive activation energy in relation to temperature. The conduction route for the enhanced transport was the serial grain boundary. In addition, the route was compared with that of nanostructured zirconia with fluorite crystal-structure.
\end{abstract}

\section{Introduction}

The high proton-conducting solids have come into focus and are being extensively studied for applications to electrochemical devices such as fuel cells, sensors, batteries, and hydrogen separators [1-3]. The proton-conducting polymers (e.g., Nafion) are commercialized owing to their high proton conductivity and low cost fabrication $[4,5]$. However, the protonic conductors still have problems with respect to swelling caused by water and deformation by heat, which are characteristics of polymers [5]. In contrast to these materials, some ceramics having perovskite structure, such as cerates and zirconates, can last against those drawbacks $[6,7]$. In particular, chemically and mechanically stable proton conductors such as barium zirconate- $\left(\mathrm{BaZrO}_{3}-\right)$ based ceramics have shown very high protonic conductivity in bulk (and in single crystals) over a wide temperature range $[3,8]$. However, the measured overall (or total) conductivity is still markedly lower than that of the bulk $[3,9,10]$. This strongly implies that the protonic resistance for grain-to-grain transfer in polycrystalline structured ceramics is significantly high. On the other hand, this suggests that one may be able to modify the overall conductivity by optimizing the properties of the grain boundary $(\mathrm{gb})$.

In the past decade, the electrical nature of the gb in nanostructured ionic conductors has attracted much attention because, in these materials, ionic transport is likely controlled by the gb [11-13]. The ionic conductivity of the nanostructured conductors would then be enhanced if the gb in these materials is more conductive than the bulk. Consequently, extensive research effort has been devoted to measuring the ionic conductivity of nanostructured ionic conductors, in particular, the nanostructured protonic conductors such as doped barium zirconate and cerate perovskites [14, 15]. However, only a few studies have reported enhanced gb conductivity in those materials [9]. Therefore, not that long ago, it was generally believed that the gb was not a preferred route for protonic conduction in the perovskites. However, it was revealed in this study that this common assumption could be false when the material was within a certain range of temperature.

In this contribution, nanostructured yttrium-doped barium zirconate $\left(\mathrm{BaZr}_{0.9} \mathrm{Y}_{0.1} \mathrm{O}_{3}, \mathrm{n}\right.$-BZY) was prepared and its grain and gb protonic conduction was investigated over a 
wide temperature range $\left(\mathrm{RT} \sim 400^{\circ} \mathrm{C}\right)$. Unexpected protonic conduction in the nanostructure (enhanced protonic conduction in the $\mathrm{gb}$ ) was observed at low temperatures. For comparison, nanostructured yttrium-doped zirconia $\left(\mathrm{Zr}_{0.84} \mathrm{Y}_{0.16} \mathrm{O}_{2}, \mathrm{n}\right.$-YSZ) was also fabricated and its electrical property was investigated.

\section{Experimental Details}

For the preparation of $\mathrm{BaZr}_{0.9} \mathrm{Y}_{0.1} \mathrm{O}_{3}$ nanopowders, solgel method was applied with the stoichiometric quantities of $\mathrm{Ba}\left(\mathrm{NO}_{3}\right)_{2}$ (>99\%, Sigma Aldrich), $\mathrm{ZrO}\left(\mathrm{NO}_{3}\right)_{2} \cdot x \mathrm{H}_{2} \mathrm{O}$ (>99\%, Sigma Aldrich), and $\mathrm{Y}\left(\mathrm{NO}_{3}\right)_{3} \cdot 6 \mathrm{H}_{2} \mathrm{O}(99.9 \%$, Sigma Aldrich) (refer to a detailed procedure [16]). The spark plasma sintering (SPS) technique was employed to obtain a sintered nanostructure pellet (n-BZY). The powders were poured in a graphite die, heated at $200^{\circ} \mathrm{C} / \mathrm{min}$ to the sintering temperature $\left(1400^{\circ} \mathrm{C}\right)$ with an applied pressure of $100 \mathrm{MPa}$, and held for $5 \mathrm{~min}$ [9]. The relative density of the sintered pellets was $\sim 94 \%$. For the nanostructured $\mathrm{Zr}_{0.84} \mathrm{Y}_{0.16} \mathrm{O}_{2}$, commercial YSZ (fuel cell materials, particle size $\sim 10 \mathrm{~nm}$ ) nanopowder was used. A similar SPS-technique was used to obtain the sintered nanostructure pellet (n-YSZ). The sintering temperature was $850^{\circ} \mathrm{C}$ for $5 \mathrm{~min}$ and the pressure applied was $600 \mathrm{Mpa}$ (see the reference that we previously reported; grain size $\sim 45 \mathrm{~nm}$ [17]). The formed phases were identified by X-ray diffraction (XRD, X'pert, Philips) using the sintered pellets. The microstructures of the pellets were examined using a scanning electron microscope (SEM, S5500, Hitachi).

The electrical resistance of the nanostructures was measured as a function of temperature $\left(T=\mathrm{RT} \sim 400^{\circ} \mathrm{C}\right)$ using 2-probe ac impedance in wet air $\left(P_{\mathrm{H}_{2} \mathrm{O}} \sim 2.3 \times 10^{-2}\right.$ atm and $\left.\mathrm{Po}_{2} \sim 0.21 \mathrm{~atm}\right)$. The resistance was also investigated with different water partial pressure $\left(P_{\mathrm{H}_{2} \mathrm{O}}: 9 \times 10^{-3} \sim 2 \times 10^{-2} \mathrm{~atm}\right)$. Pt-ink was painted on top of the sample and heated at $700^{\circ} \mathrm{C}$ for $1 \mathrm{~h}$ to serve as the current collector. Impedance spectra were obtained in the frequency range from $0.1 \mathrm{~Hz}$ to $10 \mathrm{MHz}$, using an impedance analyzer. For the fitting of the data, the Z-view program (Scribner Associates) was used.

\section{Results and Discussion}

An XRD-study was carried out to identify the barium zirconate (the sintered pellet was used) and the result is shown in Figure 1. As seen, a single barium zirconate phase with perovskite crystal structure was confirmed by matching their peak positions with the JCPDS-card of $\mathrm{BaZrO}_{3}$. The lattice parameter was $\sim 0.420 \mathrm{~nm}$, signifying that yttrium (the dopant) was doped onto $\mathrm{Zr}$-sites of the perovskite. However, the detected peaks were a little broadened, which was caused by the nanosized grains. From the peak broadening, the grain size of the pellet was computed using Scherrer's equation $\left(d_{\mathrm{avg}} \approx k \lambda / \beta \cos \theta\right)$ [18]. It was $\sim 50 \mathrm{~nm}$, which was a little less than the size computed using the linear interception method from the SEM image (the grain size $\sim 90 \mathrm{~nm}$ ). The inserted SEM image also showed that n-BZY is highly dense (the presence of few pores in the sample was seen).

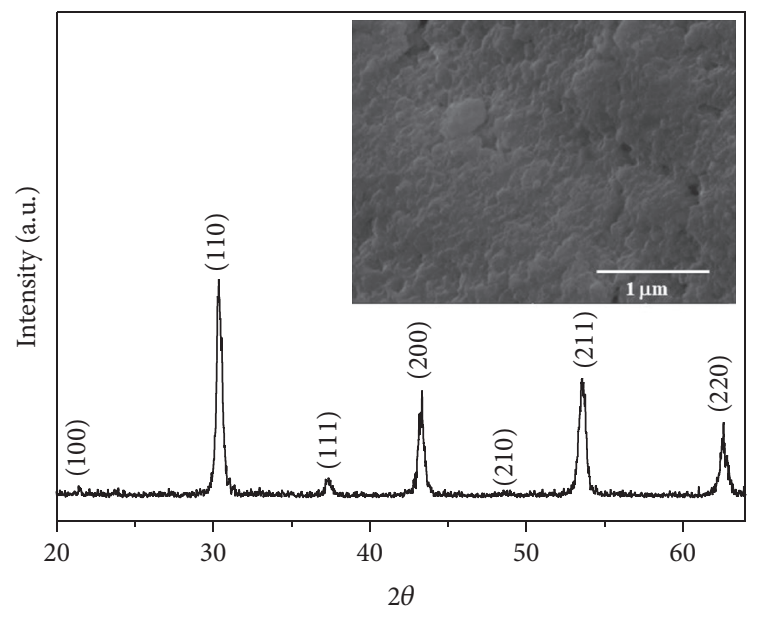

FIGURE 1: XRD pattern of $n-B Z Y$ after sintering: the inserted picture is the SEM image of the fractured n-BZY.

Figure 2 shows the representative impedance spectra of nBZY. When dry, a clear large semicircular arc was observed at the high temperature but an imperfect arc was shown at the low temperature due to high resistance. This high resistance was greatly reduced under wet conditions, such that clear semicircular arcs were observed at both temperatures. For both temperatures, the spectrum of n-BZY consisted of a large semicircular arc that partially overlapped another arc that subsequently appears at higher frequencies in wet air (the inset clearly shows the overlapped arc in the higher frequency region). The spectra obtained included two semicircular arcs, typically associated with the grain (bulk) and the grain boundary $(\mathrm{gb})$ impedance in sequence.

The resistance $(R)$ and capacitance $(C)$ of the grain and $\mathrm{gb}$ were estimated by fitting the impedance spectrum using a corresponding equivalent circuit based on a bricklayer model. Two parallel $R Q$ circuits in series were employed to fit the impedance data (i.e., $R_{\text {bulk }} Q_{\text {bulk }}$ and $R_{\mathrm{gb}} Q_{\mathrm{gb}}$ with the subscripts bulk and gb denoting the grain and the grain boundary, respectively. $Q$ denotes a constant phase element, given as $\left.C=\left(R^{1-n} Q\right)^{1 / n}\right)$. The values of $C_{\text {bulk }}$ and $C_{\mathrm{gb}}$ obtained from $\mathrm{n}$-BZY were $\sim 5 \times 10^{-12}$ and $\sim 3 \times 10^{-11} \mathrm{~F}$ at $200^{\circ} \mathrm{C}$, respectively.

Figure 3 shows the resistivity of the grain $\left(\rho_{\text {bulk }}\right)$ and $\mathrm{gb}\left(\rho_{\mathrm{gb}}\right)$ in $\mathrm{n}$-BZY. As expected from the impedance, high resistance was observed under dry air whereas it was greatly reduced under wet air, meaning that the protonic conduction was generated under wet conditions. In order to confirm the protonic conduction when wet, the grain resistivity $\left(\rho_{\text {bulk }}=\right.$ $1 / \sigma_{\text {bulk }}$, solid line) of $\mathrm{BaZr}_{0.9} \mathrm{Y}_{0.1} \mathrm{O}_{3}$ was estimated using the Nernst-Einstein relation, given as follows:

$$
\sigma_{\text {bulk }}=\frac{\left[\mathrm{OH}_{\mathrm{O}}^{*}\right] e^{2} D_{\mathrm{OH}}}{k_{B} T},
$$

where $D_{\mathrm{OH}}$ is the diffusivity of the protonic defect and $e$ and $k_{B}$ denote an elementary charge and the Boltzmann constant, respectively (for this calculation, the values of $\left[\mathrm{OH}_{\mathrm{O}}^{*}\right]$ and $D_{\mathrm{OH}}$ at different $T$ for $\mathrm{BaZr}_{0.9} \mathrm{Y}_{0.1} \mathrm{O}_{3}$ were taken 


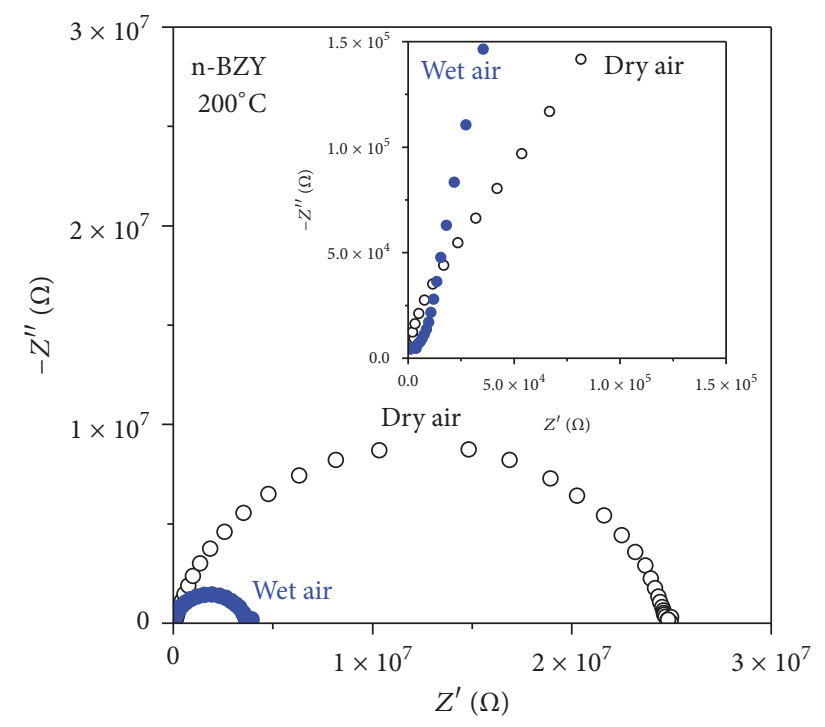

(a)

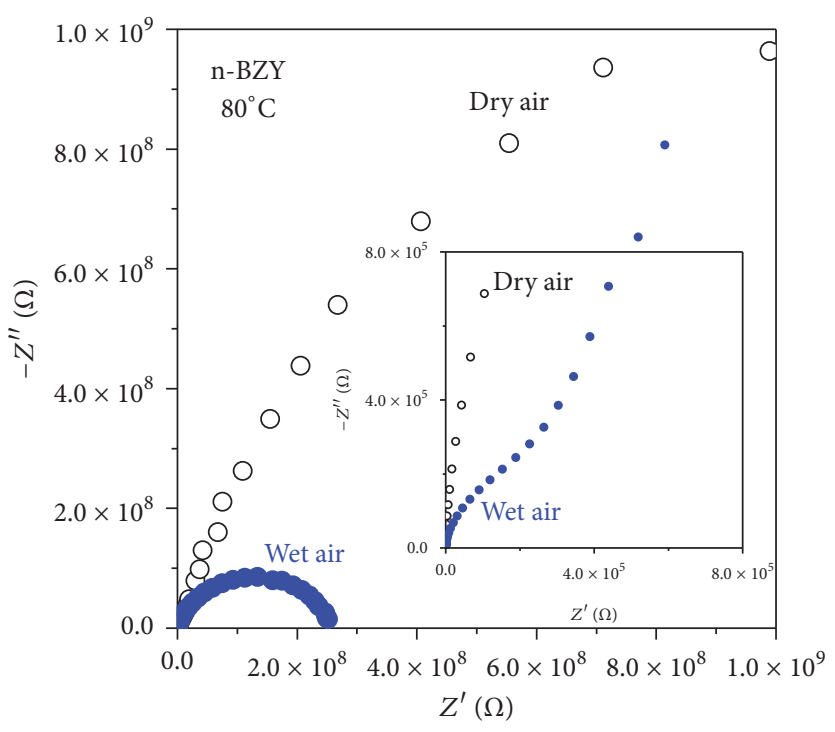

(b)

FIgURE 2: Impedance patterns of n-BZY in dry and wet air at (a) $200^{\circ} \mathrm{C}$ and (b) $80^{\circ} \mathrm{C}$. The insets show the spectra in the higher frequency region. The first and second semicircles correspond to the bulk and gb, respectively.

from the literature [3]). The measured resistivity $\left(\rho_{\text {bulk }}=\right.$ $R_{\text {bulk }} A / L$ with $L$ and $A$ being the sample thickness and the electrode area, resp.) was in quite good agreement with the estimated value (the solid line), indicating that the sample was fully equilibrated with the gaseous water and that the grain conduction was governed by the protonic defects $\left(\left[\mathrm{OH}_{\mathrm{O}}^{*}\right]\right)$ over the entire temperature range of interest.

On the other hand, the gb was not the preferred route for the protonic conduction due to high resistance, as seen in the figure. However, enhanced protonic transport was observed at low temperature $\left(<100^{\circ} \mathrm{C}\right)$ in water-saturated air, leading to dramatic reductions in the total ionic resistivity $\left(\rho_{\text {total }}\right)$ by orders of magnitude, relative to the protonic resistivity extrapolated from higher temperatures (note that this protonic conduction in the $\mathrm{n}-\mathrm{BZY}$ at low temperatures was an interfacially driven phenomenon because microstructured BZY with grain size $\sim 1 \mu \mathrm{m}$ hardly showed protonic conduction, although the data are not shown here). Similar behavior was reported in nanostructured fluorites such as zirconia and ceria [13]. In order to directly compare the electrical behavior of $\mathrm{n}$-YSZ with that of n-BZY, the electrical conduction of $n$-YSZ was also investigated and the result is presented in Figure 3. As reported, $\rho_{\text {total }}$ of n-YSZ was abruptly reduced at low temperatures (below $150^{\circ} \mathrm{C}$ ) due to the generation of protonic conduction (note that YSZ is a famous, fast oxygen ionic conductor so that its charge carriers at high temperature are oxygen ions). According to the report [13], it was also found that the source of protons at low temperatures originated from the adsorption of water on the $\mathrm{gb}$ of the fluorites and that the protons transferred through a vehicle mechanism. Thus, the protonic conduction strongly depends on the hydration and dehydration of water, showing a positive activation energy on temperature [13]. Similarly, in n-BZY, the decrease of $\rho_{\mathrm{gb}}$ when temperature fell below

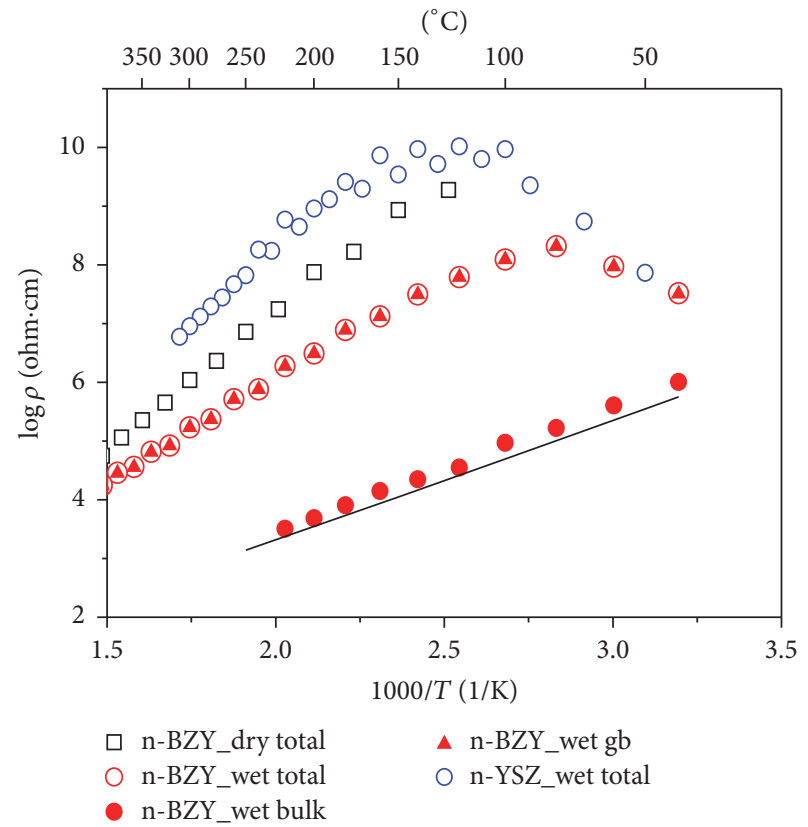

FIGURE 3: The temperature dependence of the electrical resistivity of n-BZY under dry and wet conditions. The grain, gb, and total protonic resistivities were computed from the impedance patterns. The solid line represents the estimated protonic resistivity. For comparison, the electric resistivity of n-YSZ under wet conditions was inserted.

$100^{\circ} \mathrm{C}$, a positive activation, would be due to the hydration and dehydration of water adsorbed to the gb.

Although the conducting behavior in relation to temperature was similar between n-BZY and n-YSZ, the conducting route was different. As mentioned above, protonic 


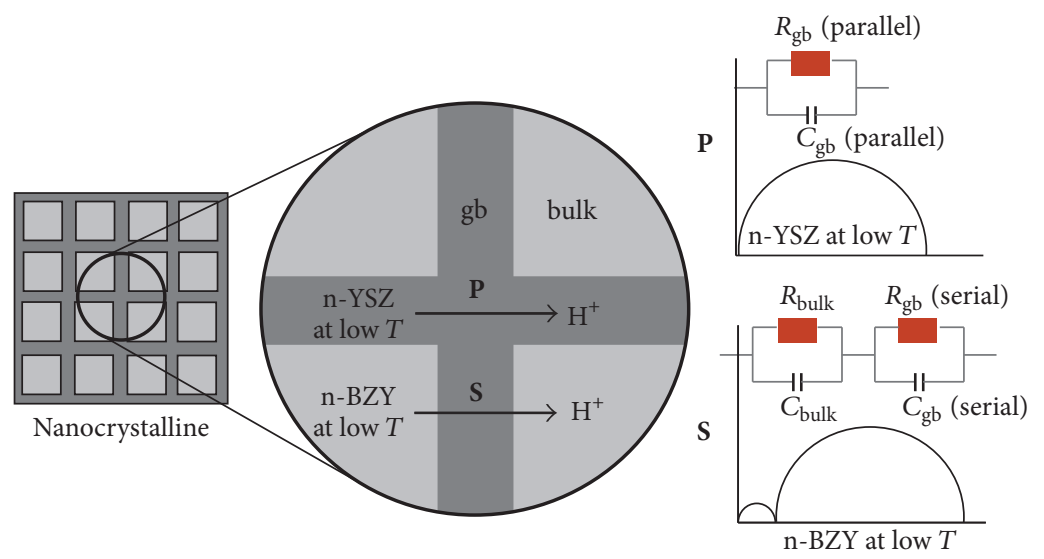

(a)

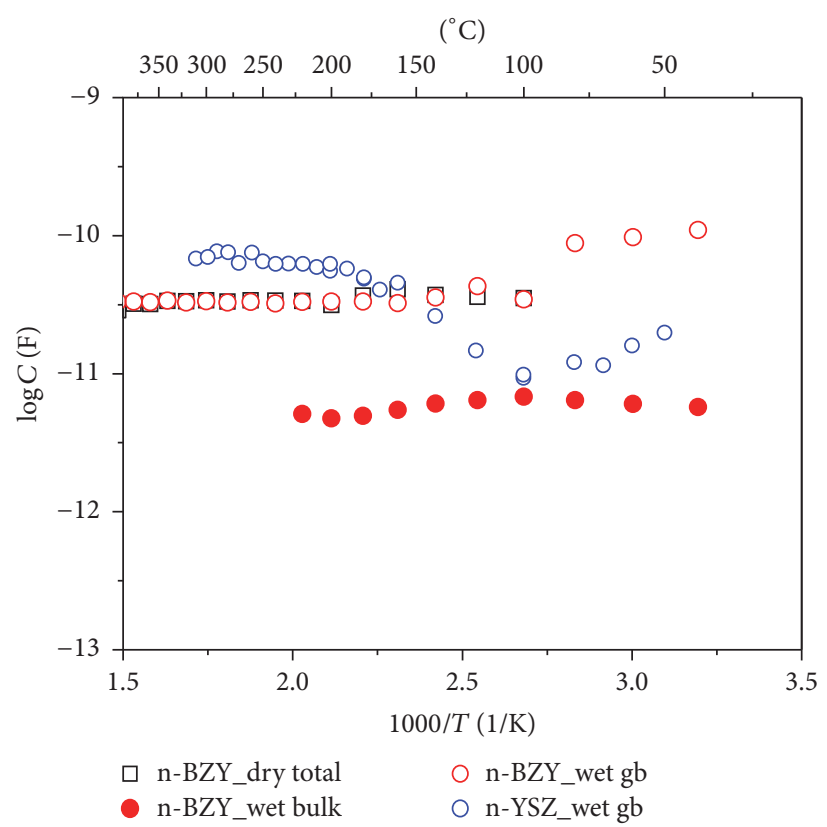

(b)

FIGURE 4: (a) Schematic diagrams of a bricklayer model for a polycrystalline ceramic and impedance spectra (Nyquist plots) expected from the current path of n-YSZ and n-BZY at low temperatures. (b) Capacitance of n-BZY measured under dry and water-saturated air, as a function of temperature. The grain boundary capacitance of $n-Y S Z$ was compared with that of n-BZY.

conduction in n-YSZ only occurs in the gb, so the grain is highly resistant to protonic conduction because YSZ is an oxygen ionic conductor. On the other hand, BZY is a protonic conductor so the grain still conducts protons, even at low temperatures. This difference leads the two to use different routes for protonic conduction. Figure 4 shows schematic diagrams of a polycrystalline current path and corresponding impedance pattern, respectively. If the current takes "P," just a single impedance pattern should be generated by a $R-C$ circuit; then the capacitance should decrease. On the other hand, if it takes "S," two impedance patterns should be made by two $R-C$ circuits and the capacitance should increase. In the n-YSZ, the current would bypass the bulk to take the parallel gb path because its $\rho_{\mathrm{gb}}$ was much lower than $\rho_{\text {bulk }}$ (the current takes "P" in Figure 4(a)). In fact, a single impedance pattern was observed in $\mathrm{n}$-YSZ at low temperatures, even though it was not shown here, and the capacitance increased as seen in Figure 4(b), implying that the protons in $n$ YSZ selectively transfer along the parallel gb. However, the current in n-BZY took " $S$ " because $\rho_{\mathrm{gb}}$ was still higher than $\rho_{\text {bulk }}$ in spite of significant reduction and, accordingly, two distinctive impedance patterns should be formed. Indeed, n-BZY generated two arcs at low temperature, as seen in Figure 2, and the capacitance measured increased as seen in Figure 4(b). This means that the protons in n-BZY transfer using route " $S$ " at low temperatures.

Although the gb transport was improved, $\rho_{\mathrm{gb}}$ was still higher than $\rho_{\text {bulk }}$ at all measured temperatures such that $\rho_{\text {total }}$ was governed by the gb transport (as seen in Figure 3), and $\rho_{\text {total }}$ was $\sim 3 \times 10^{7} \Omega \mathrm{cm}$ at room temperature. It is noteworthy that it was similar to the $\rho_{\text {total }}$ of n-YSZ with smaller grain size. According to the report, the interfacial protonic conduction 


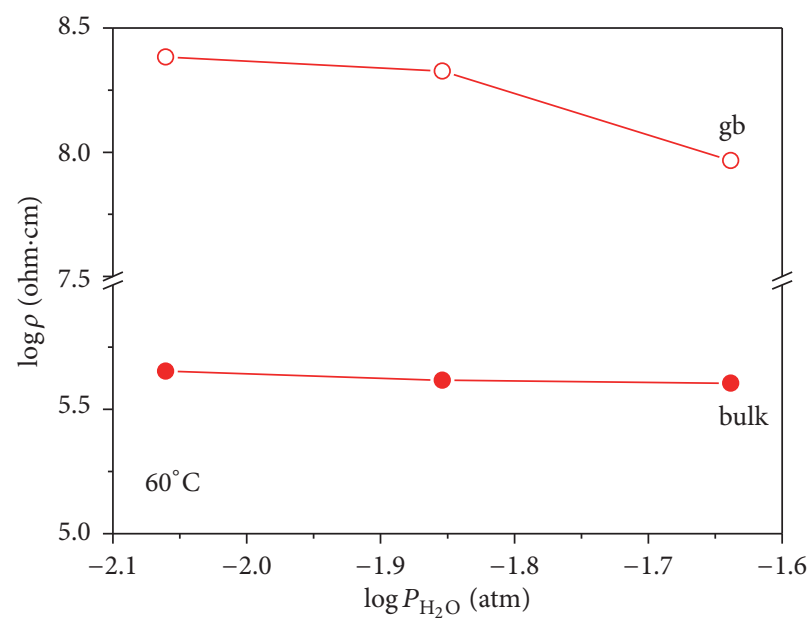

(a)

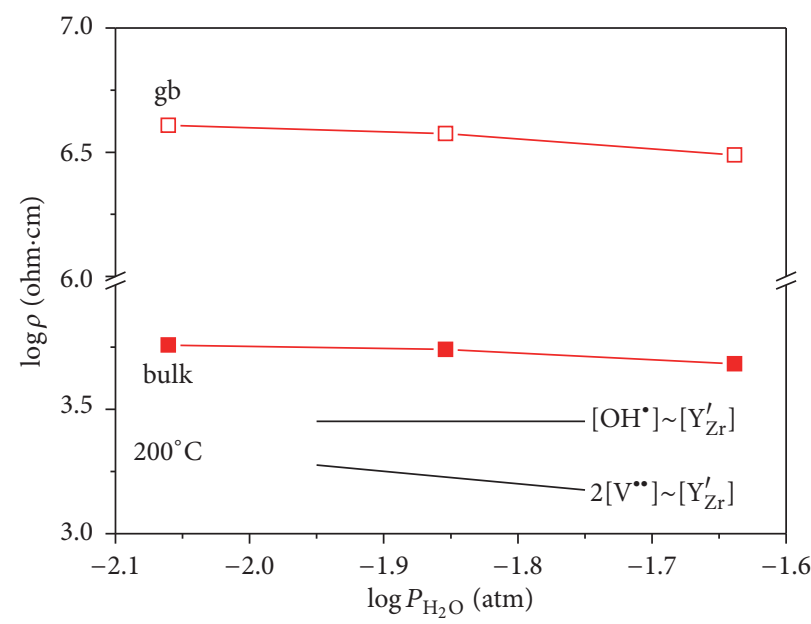

(b)

FIGURE 5: The water partial pressure $\left(P_{\mathrm{H}_{2} \mathrm{O}}\right)$ dependence of the grain and gb resistivity at (a) low and (b) high temperatures, respectively.

in nanostructured fluorites became noticeably high when the grain was smaller than $100 \mathrm{~nm}$ and increased dramatically with decreasing grain size [13]. Thus, if the grain size of n-BZY were reduced, the protonic conduction would be enhanced more at low temperatures, which could increase the potential of solid electrolytes in low-temperature fuel cells.

Figure 5 shows the $P_{\mathrm{H}_{2} \mathrm{O}}$ dependence of the protonic resistivity in n-BZY. The solid and open symbols represent the bulk and gb protonic resistivity, respectively. As can be seen at low temperature, $\rho_{\text {bulk }}$ weakly depended on $P_{\mathrm{H}_{2} \mathrm{O}}$ while $\rho_{\mathrm{gb}}$ was more sensitive to $P_{\mathrm{H}_{2} \mathrm{O}}$. Such strong dependency of the gb was due to the hydration and dehydration mentioned above. On the other hand, at high temperature, both the grain and gb protonic conduction slightly depended on $P_{\mathrm{H}_{2} \mathrm{O}}$. The dependency was between zero and $1 / 2$, which was understandable considering the defect chemistry of doped barium zirconate. Such dependency was observed in the case that the electroneutral condition (ENC) was $2\left[\mathrm{~V}_{\mathrm{O}}^{* *}\right]+$ $\left[\mathrm{OH}_{\mathrm{O}}^{*}\right]=\left[\mathrm{Y}_{\mathrm{Zr}}^{\prime}\right]$, where $\left[\mathrm{V}_{\mathrm{O}}^{*}\right]$ and $\left[\mathrm{Y}_{\mathrm{Zr}}^{\prime}\right]$, approximately, are the concentration of the oxygen vacancy and dopant, respectively (details are described in [10]).

\section{Conclusion}

Nanostructured yttrium-doped barium zirconate was prepared using spark plasma sintering; then its grain and grain boundary electrical properties were measured separately over a wide range of temperature in wet and dry air. Both grain and grain boundary protonic transport was observed under these conditions. The grain protonic resistivity matched well the estimated value, showing that it was governed by the concentration of the protonic defects. The gb highly resisted protonic transport, compared to the grain; however, enhanced protonic grain boundary conduction was observed at certain temperatures $\left(<100^{\circ} \mathrm{C}\right)$. The conduction pathway for the enhanced conduction was analyzed using the impedance pattern and capacitance value. It was found that it was a serial grain boundary. At low temperatures, the grain boundary resistivity depended greatly on the partial pressure of water.

\section{Competing Interests}

The authors declare that there is no conflict of interests regarding the publication of this paper.

\section{Acknowledgments}

The authors thank D. W. Jung for sample preparation and S. Kim for valuable discussion. This research was supported by the Daejeon University fund (201501400001).

\section{References}

[1] G. Alberti and M. Casciola, "Solid state protonic conductors, present main applications and future prospects," Solid State Ionics, vol. 145, no. 1-4, pp. 3-16, 2001.

[2] H. Iwahara, "Technological challenges in the application of proton conducting ceramics," Solid State Ionics, vol. 77, no. 1-2, pp. 289-298, 1995.

[3] K. D. Kreuer, "Proton-conducting oxides," Annual Review of Materials Research, vol. 33, pp. 333-359, 2003.

[4] F. M. Vichi, M. T. Colomer, and M. A. Anderson, "Nanopore ceramic membranes as novel electrolytes for proton exchange membranes," Electrochemical and Solid-State Letters, vol. 2, no. 7, pp. 313-316, 1999.

[5] Y. M. Li, M. Hibino, M. Miyayania, and T. Kudo, "Proton conductivity of tungsten trioxide hydrates at intermediate temperature," Solid State Ionics, vol. 134, no. 3-4, pp. 271-279, 2000.

[6] M. J. Scholten, J. Schoonman, J. C. van Miltenburg, and H. A. J. Oonk, "Synthesis of strontium and barium cerate and their reaction with carbon dioxide," Solid State Ionics, vol. 61, no. 1-3, pp. 83-91, 1993.

[7] N. Bonanos, K. S. Knight, and B. Ellis, "Perovskite solid electrolytes: structure, transport properties and fuel cell applications," Solid State Ionics, vol. 79, pp. 161-170, 1995. 
[8] H. Iwahara, T. Yajima, T. Hibino, K. Ozaki, and H. Suzuki, "Protonic conduction in calcium, strontium and barium zirconates," Solid State Ionics, vol. 61, no. 1-3, pp. 65-69, 1993.

[9] H. J. Park, Z. A. Munir, and S. Kim, "Grain boundary protonic conductivity in highly dense nano-crystalline Y-doped $\mathrm{BaZrO}_{3}$," Journal of the Korean Ceramic Society, vol. 47, no. 1, pp. 71-74, 2010.

[10] H. J. Park, "Electrical properties of the protonic conductor 1 mol\% Y-doped $\mathrm{BaZrO}_{3}$," Journal of Solid State Electrochemistry, vol. 15, pp. 2205-2211, 2011.

[11] Y.-M. Chiang, E. B. Lavik, I. Kosacki, H. L. Tuller, and J. Y. Ying, "Defect and transport properties of nanocrystalline CeO2-x," Applied Physics Letters, vol. 69, no. 2, pp. 185-187, 1996.

[12] M. Shirpour, G. Gregori, R. Merkle, and J. Maier, "On the proton conductivity in pure and gadolinium doped nanocrystalline cerium oxide," Physical Chemistry Chemical Physics, vol. 13, no. 3, pp. 937-940, 2011.

[13] S. Miyoshi, Y. Akao, N. Kuwata et al., "Low-temperature protonic conduction based on surface protonics: an example of nanostructured yttria-doped zirconia," Chemistry of Materials, vol. 26, no. 18, pp. 5194-5200, 2014.

[14] R. B. Cervera, Y. Oyama, S. Miyoshi, K. Kobayashi, T. Yagi, and S. Yamaguchi, "Structural study and proton transport of bulk nanograined Y-doped $\mathrm{BaZrO}_{3}$ oxide protonics materials," Solid State Ionics, vol. 179, no. 7-8, pp. 236-242, 2008.

[15] X. Ma, J. Dai, H. Zhang, and D. E. Reisner, "Protonic conductivity nanostructured ceramic film with improved resistance to carbon dioxide at elevated temperatures," Surface \& Coatings Technology, vol. 200, no. 5-6, pp. 1252-1258, 2005.

[16] S. N. A. Bakar, I. A. Talib, and N. Osman, "The effect of different molar ratio citric acid and ethylene glycol to metal cation on the ceramics powder of $\mathrm{Y}^{3+}$ doped $\mathrm{BaZrO}_{3}$," Solid State Science and Technology, vol. 69, no. 2, pp. 36-44, 2010.

[17] H. J. Park and Y. H. Choa, "The grain boundary conduction property of highly dense and nanostructured yttrium-doped zirconia," Electrochemical and Solid-State Letters, vol. 13, no. 5, pp. K49-K52, 2010.

[18] L. Alexander and H. P. Klug, "Determination of crystallite size with the X-ray spectrometer," Journal of Applied Physics, vol. 21, no. 2, pp. 137-142, 1950. 

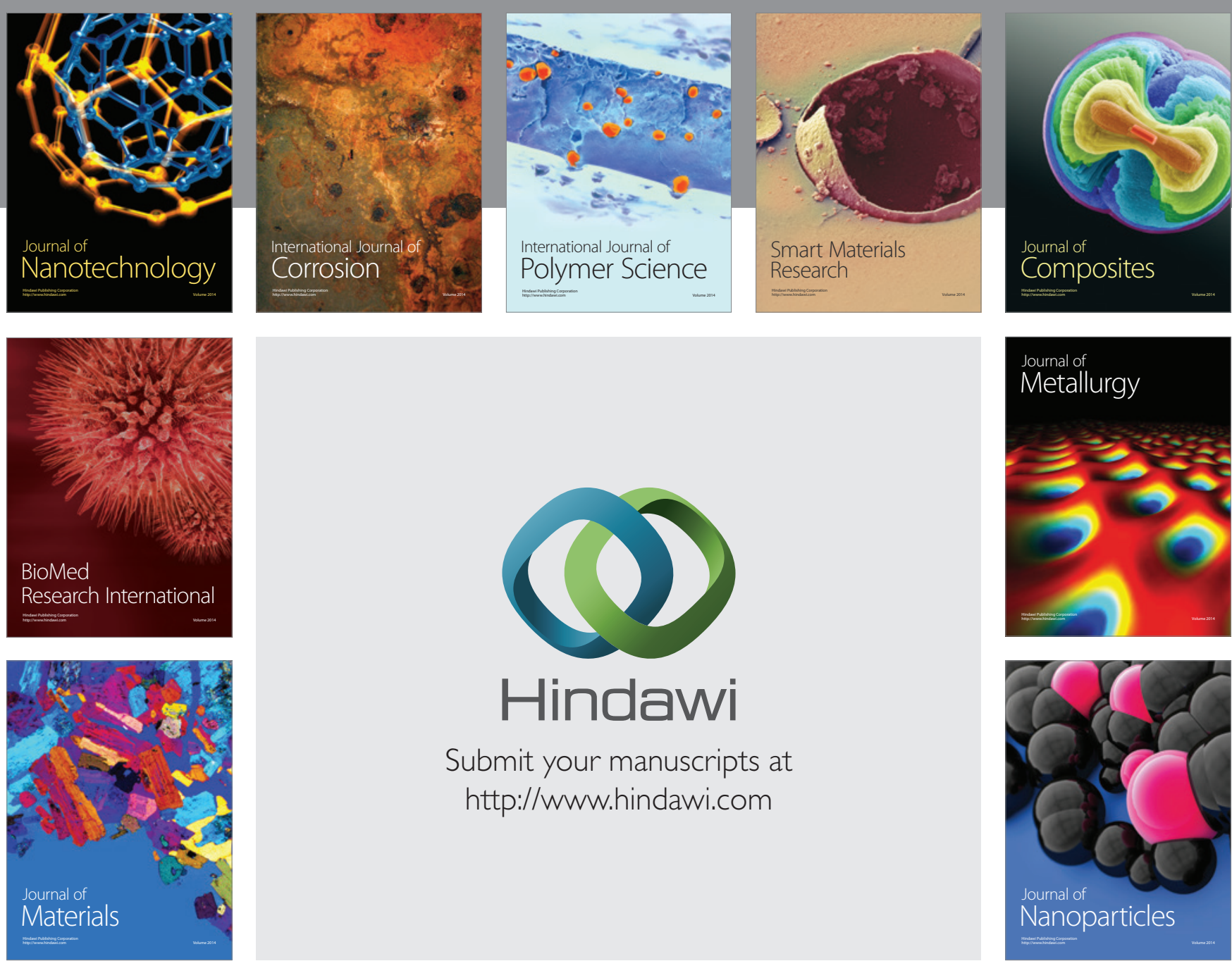

\section{Hindawi}

Submit your manuscripts at

http://www.hindawi.com

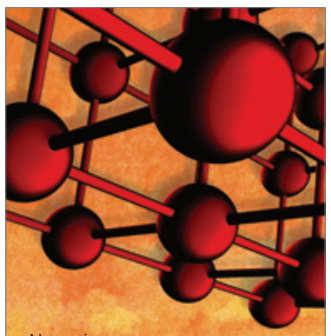

Materials Science and Engineering
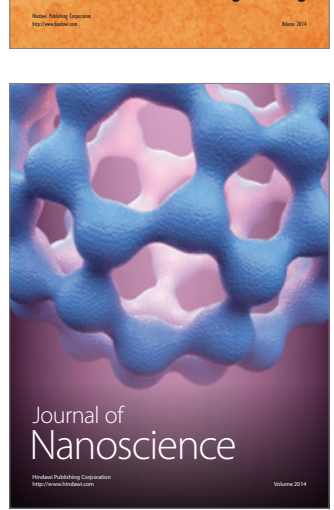
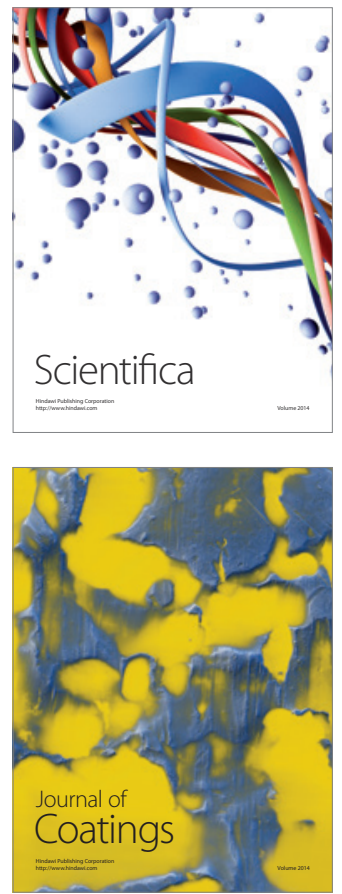
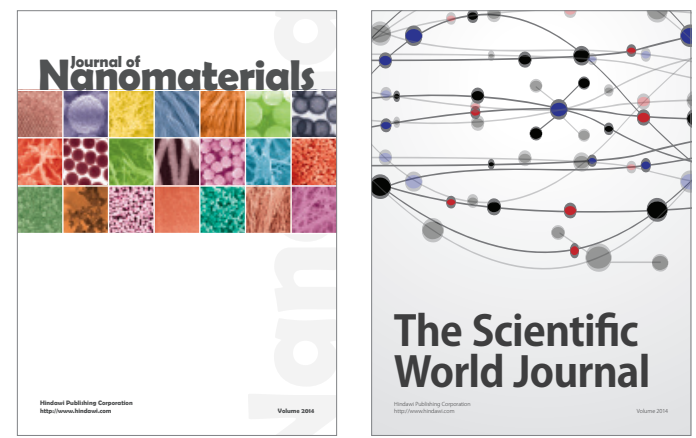

The Scientific World Journal
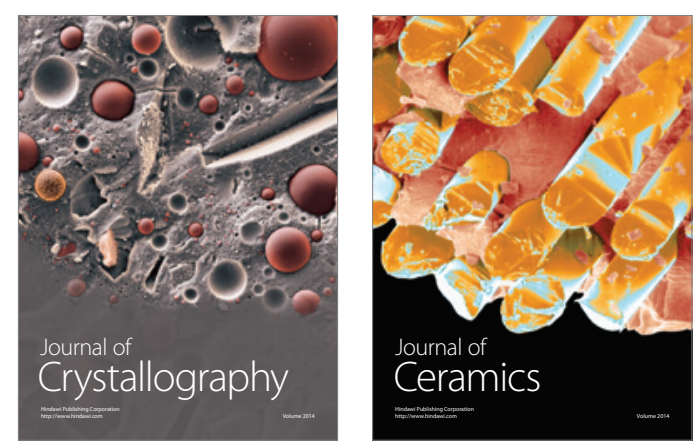
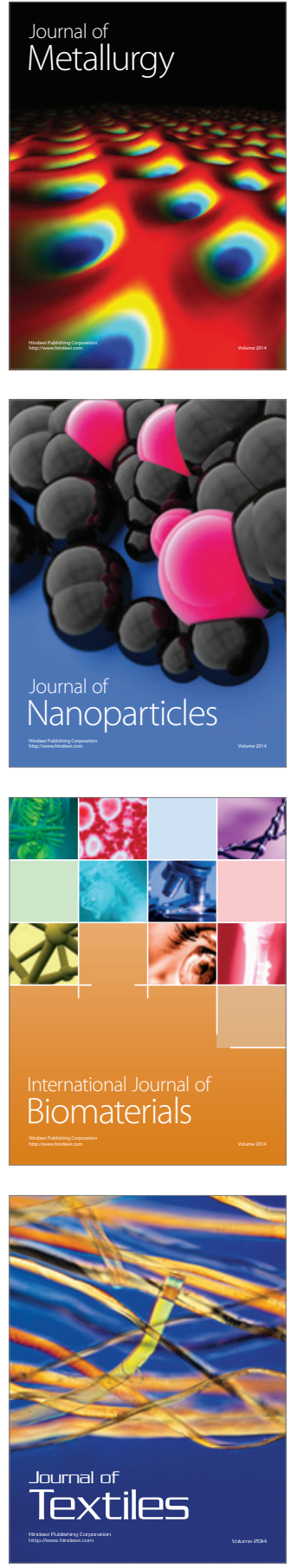